\title{
On persistently positively expansive maps
}

\author{
ALEXANDER ARBIETO \\ Instituto de Matemática, Universidade Federal do Rio de Janeiro, Caixa Postal 68530, 21945-970 Rio de Janeiro, RJ, Brasil \\ Manuscript received on May 19, 2009; accepted for publication on September 30, 2009
}

\begin{abstract}
In this paper, we prove that any $C^{1}$-persistently positively expansive map is expanding. This improves a result due to Sakai (Sakai 2004).
\end{abstract}

Key words: positively expansive maps, expanding maps.

\section{INTRODUCTION}

Let $M$ be a compact Riemannian manifold. We say that a continuous map $f: M \rightarrow M$ is $c$-positively expansive for some $c>0$ if, for any $x \neq y$, there exists $n \geq 0$, such that $d\left(f^{n}(x), f^{n}(y)\right) \geq c$. These maps were studied by many authors, see for instance (Coven and Reddy 1980), (Ruelle 1978), (Sakai 1985) and (Sakai 2003). An important class of positively expansive maps are the expanding ones defined as follows: we say that a map is expanding if there are constants $C>0$ and $\sigma>1$, such that $\left\|D f^{n}(x) v\right\| \geq C \sigma^{n}\|v\|$ for every $x \in M$.

Definition 1.1. We say that a $C^{1}$ map $f: M \rightarrow M$ is $C^{1}$-persistently positively expansive map if there exists a $C^{1}$-neighborhood $\mathcal{U}$, such that for any $g \in \mathcal{U}$, there exists $c(g)>0$, such that $g$ is $c(g)$ positively expansive.

In this note, we shall prove the following result:

THEOREM 1.2. Let $M$ be a compact manifold. Any $C^{1}$-persistently positively expansive map $f: M \rightarrow M$ is expanding.

The same type of result appears in (Sakai 2004). However, Sakai assumes that the separation property in the definition of positive expansivity holds for any composition of maps in the neighborhood $\mathcal{U}$, and, in his paper, $c(g)$ is also a constant in the neighborhood. Since we are not assuming these hypotheses, our result is slightly stronger. Also, our arguments rely on a theorem by Cao (Cao 2003), which simplifies the proof. In what follows, we recall the results of Sakai (Sakai 2004).

2000 AMS Subject Classification: 37D20 (Primary), 37C50 (Secondary).

E-mail: arbieto@im.ufrj.br 
In Sakai's paper, the following definition is introduced: a map $f$ is a $C^{1}$-stably positively expansive map with constants $v>0$ and $c>0$ if, for any sequence, $\left\{g_{i}\right\}_{i=1}^{\infty} \subset C^{1}(M)$, with $d_{C^{1}}\left(f, g_{i}\right) \leq v$ for all $i \in \mathbb{N}$ and for any sequences $\left\{x_{i}, y_{i}\right\}_{i=0}^{\infty}$, such that $x_{i}=g_{i} \circ \cdots \circ g_{1}\left(x_{0}\right), y_{i}=g_{i} \circ \cdots \circ g_{1}\left(y_{0}\right)$ and $d\left(x_{i}, y_{i}\right) \leq c$; for all $i \geq 0$, we have that $x_{0}=y_{0}$. Then, he proves that any $C^{1}$-stably positively expansive map is expanding.

\section{PROOF OF THE THEOREM}

First, we recall a lemma due to Franks (Franks 1971):

LEMMA 2.1. Let $f$ be a $C^{1}$ map and $\mathcal{U}$ a $C^{1}$-neighborhood of $f$. Then, there exists a neighborhood $\mathcal{U}_{0} \subset \mathcal{U}$ of $f$ and $a \delta>0$ such that, if $g \in \mathcal{U}_{0}(f), S=\left\{p_{1}, \ldots, p_{m}\right\} \subset M$ is a finite set, and $\left\{L_{i}: T_{p_{i}} M \rightarrow\right.$ $\left.T_{g\left(p_{i}\right)} M\right\}_{i=1}^{m}$ are linear maps satisfying $\left\|L_{i}-D g\left(p_{i}\right)\right\| \leq \delta$ for $i=1, \ldots m$; then, there exists $h \in \mathcal{U}_{0}(f)$ satisfying $h\left(p_{i}\right)=g\left(p_{i}\right)$ and $D h\left(p_{i}\right)=L_{i}$. Moreover, if $U$ is a neighborhood of $S$, then, $h$ can be taken such that $h(x)=g(x)$ for every $x \in S \cup(M-U)$, and locally

$$
h(x)=\exp _{g\left(p_{i}\right)} \circ D g\left(p_{i}\right) \circ \exp _{p_{i}}^{-1}(x), \text { for } x \in B_{\varepsilon}\left(p_{i}\right),
$$

where exp is the exponential map of the Riemannian manifold $M$, and $\varepsilon$ is sufficiently small.

Now we fix $f$ a $C^{1}$-persistently positively expansive map.

The following corollary is an adaptation of an argument of (Sakai 2004):

COROLLARY 2.2. Let $\mathcal{U}$ be the neighborhood of $f$ in the definition of $C^{1}$-persistently positively expansive map. For any $g \in \mathcal{U}$, and $p$ as a periodic point for $g$ with period $n$, if $\lambda$ is an eigenvalue of $\operatorname{Dg}^{n}(p)$, then $|\lambda|>1$.

Proof. If not, there exists $g$ with $p$ as a periodic point with a contracting eigenvalue, i.e., an eigenvalue $\lambda$ with $|\lambda| \leq 1$. Using Franks' lemma, we can find $h \in \mathcal{U}$, such that:

$$
h(x)=\exp _{g^{i}(p)} \circ D g\left(g^{i-1}(p)\right) \circ \exp _{g^{i-1}(p)}^{-1}(x), \text { for } x \in B_{\varepsilon}\left(g^{i-1}(p)\right),
$$

where $\varepsilon$ is small. Now, since we linearized near the periodic orbit, we obtain two distinct, but close enough, orbits $z$ and $w$ inside the stable local manifold of this linear map, such that $d\left(h^{n}(x), h^{n}(y)\right) \leq c(h)$ for $n \geq 0$, which is a contradiction.

The same argument shows that any $g \in \mathcal{U}$ is a local diffeomorphism. Since there exists $p \in M$ and a zero eigenvalue of $D g(p)$, by Franks' lemma, we make a perturbation $h$ with a set of points $S$ (in the direction of the eigenvalue) such that, for every $z \in S$, we have $h(z)=h(p)$, and this contradicts the positive expansivity of $h$.

A $\delta$-pseudo orbit $\left\{x_{i}\right\}$ for $f$ is a sequence of points in $M$ such that, for every $i$, we have $d\left(f\left(x_{i}\right), x_{i+1}\right)<$ $\delta$. We say that $f$ has the shadowing property if, for every $\varepsilon>0$, there exists $\delta>0$ such that, for any $\delta$-pseudo orbit for $f$, there exists $y \in M$, such that $d\left(f^{i}(y), x_{i}\right)<\varepsilon$ for all $i \geq 0$. We say that $y$ is a shadow for the pseudo-orbit. 
Since $f$ is a local diffeomorphism, in particular it is open. Now, (Ruelle 1978) and (Sakai 2003) show that any positively expansive map that is open has the shadowing property. Also, since the map is positively expansive, the shadow is unique. In particular, if the pseudo-orbit is periodic, the shadow is a periodic point.

We recall that, for any $x \in M$ and $v \in T_{x} M$, the latter being a nonzero vector, the Lyapunov exponent associated is:

$$
\lambda(x, v)=\lim _{k \rightarrow \infty} \frac{1}{k} \log \left\|D f^{k}(x) v\right\|
$$

whenever this limit exists. By Oseledets' theorem (Oseledets 1968), this limit exists for $\mu$-almost every point $x$ and any nonzero vector $v \in T_{x} M$, if $\mu$ is a finite invariant measure.

LEMMA 2.3. Let $\mu$ be a finite invariant measure of $f$. Then, for $\mu$-almost every $x$, the Lyapunov exponents $\lambda_{i}(f, x)$ are positive.

Proof. Fix $\delta>0$ as the constant given by Franks' lemma. Let $v>0$, such that, if $d(x, y)<v$, then $d(D f(x), D f(y))<\delta$. Finally, fix $\varepsilon>0$ as the constant given by the shadowing property associated to $v$-pseudo orbits.

If the lemma is false, there exists a measure $\mu$ with a non-positive Lyapunov exponent. By Poincaré recurrence, there exists $x \in M, v \in T_{x} M, 0<\eta \ll \delta^{1}$ and $N$ large enough, such that:

$$
\left\|D f^{N}(x) v\right\| \leq(1+\eta)^{N}\|v\|, \quad d\left(f^{N}(x), x\right)<v,
$$

and there exists $i$ much smaller than $N$, such that $d\left(f^{i}(x), x\right)<v$ and the directions

$$
\frac{D f^{i}(x) v}{\left\|D f^{i}(x) v\right\|} \quad \text { and } \quad \frac{D f^{N}(x) v}{\left\|D f^{N}(x) v\right\|}
$$

are close enough.

The shadowing lemma implies that there exists a periodic orbit $f^{N-i}(p)=p$, such that $d\left(f^{j}(x)\right.$, $\left.f^{j}(p)\right)<v$ for $j=0, \ldots N-i-1$; moreover, there exists some vector $w \in T_{p} M-\{0\}$ close to $D f^{N-i}(p) w$. Since $\eta$ is small, we can use Frank's lemma to perturb $f$, replacing the derivative in the orbit of $p$ by the derivative of the segment of orbit of $x$, dividing by $1+2 \eta$ on each iterate, and compose the last derivative (i.e., the last iterate of the periodic orbit) with a small rotation. It was obtained $g \in \mathcal{U}$ with a periodic point $p$ of period $N-i$ with a contractive eigen- vector $w$ for the derivative $D f^{N-i}(p)$. This contradicts the previous lemma.

Now we invoke a theorem due to Cao (Cao 2003):

THEOREM 2.4 (Cao). Let $f: M \rightarrow M$ be a $C^{1}$-local diffeomorphism. If the Lyapunov exponents of every $f$-invariant probability measure are positive, then $f$ is uniformly expanding.

Together with the previous lemma, this completes the proof.

\footnotetext{
${ }^{1} \eta$ is very small compared with $\delta$.
} 


\section{ACKNOWLEDGMENTS}

The author would like to thank Professor C. Morales by useful conversations. The author would also like to thank the useful comments of an anonymous referee that clarified the proof of lemma 2.3. This work was partially supported by Conselho Nacional de Desenvolvimento Científico e Tecnológico (CNPq) and Programa de Apoio a Projetos Institucionais com a Participação de Recém-Doutores - Coordenação de Aperfeiçoamento de Pessoal de Nível Superior (PRODOC-CAPES).

\section{RESUMO}

Neste artigo, mostramos que todo mapa $C^{1}$-persistentemente positivamente expansivo é expansor. Isto melhora um resultado devido a Sakai (Sakai 2004).

Palavras-chave: mapas positivamente expansivos, mapas expansores.

\section{REFERENCES}

CAO Y. 2003. Non-zero Lyapunov exponents and uniform hyperbolicity. Nonlinearity 16: 1473-1479.

Coven E And Reddy W. 1980. Positively Expansive maps of compact manifolds. Lecture Notes in Math 819: 96-110.

FRANKS J. 1971. Necessary conditions for stability of diffeomorphisms. Trans Amer Math Soc 158: 301-308.

RUELLE D. 1978. Thermodynamical Formalism. Encyclopedia Math Appl n. 5.

SAKAi K. 1985. Periodic Points of Positively Expansive maps. Proc Amer Math Soc 94: 531-534.

SAKAI K. 2003. Various shadowing properties for positively expansive maps. Topology Appl 131: 15-31.

SAKAI K. 2004. $C^{1}$-Stably Positively Expansive Maps. Bull of the Polish Acad Sci Math 52: 197-209.

OSELEDETS V. 1968. A multiplicative ergodic theorem: Lyapunov characteristic numbers for dynamical systems. Trans Moscow Math Soc 19: 197-231. 